\title{
Demonstration of the ascending aorta in infective endocarditis by intravenous digital subtraction angiography
}

Sir,

We read with interest the article by Hunter et al (1988;60:252-8).

There seems to be some confusion in published reports about the terminology of the aortic root complications encountered in infective endocarditis. An abscess is an infective cavity that is completely enclosed, with no communication with the intravascular space. A mycotic aneurysm, however, is defined as a cavity that does communicate with the vascular space, and it usually results from the rupture of an abscess cavity into the vascular space. Further rupture into a second but different vascular space results in the formation of an infective fistula. The terminology of these lesions is important because only mycotic aneurysms and fistulas can potentially be shown by angiographic techniques, including intravenous digital subtraction angiography. Abscess cavities would be missed by both these techniques. The four cases reported in this article are therefore mycotic aneurysms and not endocarditis abscesses, as described.

Hunter et al have also overlooked the importance of echocardiography in the diagnosis of aortic root involvement by infective endocarditis. We accept that precordial cross sectional echocardiography is unreliable in the diagnosis of aortic root abscesses and mycotic aneurysms, especially when prosthetic valves are present. Transoesophageal cross sectional echocardiography, because of its improved image quality and resolution, has recently proved more sensitive in the diagnosis of these complications than precordial echocardiography..$^{1-3}$ The addition of colour flow mapping also provides important information because it can distinguish between abscess cavities and mycotic aneurysms by showing flow entering or exiting the cavity in question. Colour flow mapping is also a good diagnostic technique for showing the presence and precise delineation of any intracardiac infective fistulas.

Transoesophageal echocardiography is a safe procedure, even in acutely ill patients, which has been in routine diagnostic use in over 700 cases at the Thoraxcentre since 1985. Our experience using this technique in a wide range of patients with infective endocarditis is that it increases the diagnosis of aorti $\vec{E}$ root complications compared with precordial echocardiography. We have noted only one area where the usefulness of transoesophageal echocars diography is limited, and that is in the detection of anterior aortic root cavities in patients with aortice prostheses. These may be missed by the transoesos phageal approach because of the image "masking effect" of the prosthetic valve. In these cases the anterior cavities are usually visualised by the precoro dial approach.

In view of our experience and that of others, ${ }^{23}$ w recommend that transoesophageal echocardiograph is mandatory in all patients with suspected endocars ditis of a native or prosthetic aortic valve because it is the best diagnostic method for the infective complications.

John H Smyllie,

George R Sutherland,

Jos R T C Roelandt,

Thoraxcentre, Ba302,

Academic Hospital-Dijkzigt,

Rotterdam, The Netherlands.

\section{References}

1 Gussenhoven EJ, van Herverden LA, Roelandt J, BoN $E$, de Jong $\mathbf{N}$. Detailed analysis of aortic valve्d endocarditis: comparison of precordial, esophageal and epicardial two-dimensional echocardiograph with surgical findings. JCU 1986;14:209-11.

2 Drexler M, Erbel R, Rohmann S, Mohr-Kahaly SD Meyer J. Diagnostic value of two-dimensional transesophageal versus transthoracic echocardiography i卂 patients with infective endocarditis. Eur Heart 9 1987;8 (suppl I):303-6.

3 Daniel WG, Schroeder E, Moegge A, Lichtlen PR Transesophageal echocardiography in infective en docarditis. Am J Cardiac Imaging 1988;2:78-85. 